\title{
Magnetic phase transition in disordered Fe-Ni alloys studied by means of small-angle neutron scattering and three-dimensional analysis of the neutron depolarization
}

\author{
S. V. Grigoriev, ${ }^{1, *}$ S. A. Klimko, ${ }^{1}$ W. H. Kraan, ${ }^{2}$ S. V. Maleyev, ${ }^{1}$ A. I. Okorokov, ${ }^{1}$ M. Th. Rekveldt, ${ }^{2}$ and V. V. Runov ${ }^{1}$ \\ ${ }^{1}$ Petersburg Nuclear Physics Institute, Gatchina, St. Petersburg 188350, Russia \\ ${ }^{2}$ Interfacultair Reactor Instituut, TU-Delft, $2629 \mathrm{JB}$ Delft, The Netherlands
}

(Received 7 September 2000; revised manuscript received 8 May 2001; published 14 August 2001)

\begin{abstract}
The magnetic phase transition in iron-nickel fcc alloys $\mathrm{Fe}_{70} \mathrm{Ni}_{30}$ doped by carbon ( $0.7 \%$ at.) is investigated using the three-dimensional analysis of the neutron depolarization (ND) and small-angle neutron scattering (SANS). The coexistence and the growth of two different scale magnetic correlations are observed in a paramagnetic phase as temperature closes to $T_{C}$. The usual critical fluctuations of a Lorentzian shape with size $R_{c}<200 \AA$ are found by the analysis of SANS intensity $I(q)$. The ND analysis shows also presence of the large scale correlations (about $10^{3}-10^{4} \AA$ ) with the "squared" Lorentzian shape. We attribute these large scale correlations to local variations of the Curie temperature $T_{C}$. The local $T_{C}$ variations are described by the disorder parameters of the system: $\left\langle T_{C}\right\rangle$, a spread of $T_{C}$ variations $\Delta T_{C}$, and characteristic size of the local areas $R_{0}$, where $T_{C}$ variations occur. The ratio between the depth of the $T_{C}$ variations $\left(\Delta T_{C} /\left\langle T_{C}\right\rangle\right)$ and its characteristic correlation length $R_{0} / a$ ( $a$ is a lattice constant) determines a scenario of the transition: "percolative" or "homogeneous" ones. At $R_{0} / a \gg\left(\Delta T_{C} /\left\langle T_{C}\right\rangle\right)^{-2 / 3}$ locally ordered ferromagnetic regions appear in paramagnetic phase and form a large percolative cluster. In this case the connectivity length of the ordered regions dominates at the correlation length of thermal fluctuations. Therefore this transition goes in accordance with the "percolative" scenario. All three parameters of the transition are obtained from the data of the three dimension analysis of the ND. From the temperature dependence of the magnetization, we found $\left\langle T_{C}\right\rangle=397$ $\pm 0.5 \mathrm{~K}$ and $\Delta T_{C}=4.55 \pm 0.05 \mathrm{~K}$. From ND data we estimated the temperature independent characteristic size $R_{0}$ of the $T_{C}$ variations. It is equal to $10^{4} \AA$.
\end{abstract}

DOI: $10.1103 /$ PhysRevB.64.094426

PACS number(s): 75.50.Bb, 61.12.Ex, 75.40.-s

\section{INTRODUCTION}

The problem of a coexistence of two length scales for the fluctuations of the order parameter above the phase transition temperature $T_{C}$ has been extensively discussed for twelve years, ever since Andrews reported a line shape consisting of two components in the critical $\mathrm{x}$-ray scattering associated with the structural phase transition in $\mathrm{SrTiO}_{3}{ }^{1}$ The large scale fluctuations, present along with the critical fluctuations of an ordinary lengthscale, are attributed to some kind of disorder in the system and could be connected with quenching of the long-range disorder. ${ }^{2}$

Similarly, we established the coexistence of two different spatial scale lengths in the magnetic correlations in invar FeNi alloys above $T_{C}$ by means of a neutron depolarization (ND) and small-angle neutron scattering (SANS) measurements. ${ }^{3,4}$ It was shown in Refs. 3,4 using SANS that the smaller scale length is attributed to the critical fluctuations and their shape is well described by a Lorentzian in momentum transferred space. Using neutron depolarization (ND) much larger magnetic inhomogeneities were found above $T_{C}$. Their shape could be roughly modeled by a "squared Lorentzian" expression. Later on, ordinary ND was replaced by three-dimensional analysis of the ND in order to study how these large magnetic inhomogeneities near $T_{C}$ appear.

In the present paper we continue the investigation of the ferromagnetic phase transition in a disordered FeNi alloy. In homogeneous ferromagnets the phase transition is of second order. However, spin lattice interaction leads to a first order transition. This change of type of the transition should be strongly enhanced in the Invar by the combination of strong spin lattice interaction and disorder. On the other hand, it is well known that point defects do not change the type of the transition. Meanwhile large scale disorder leads to spatial fluctuations of the transition temperature as was pointed out by Ginzburg. ${ }^{5}$ These local $T_{C}$ variations are described by average Curie temperature $\left\langle T_{C}\right\rangle$, a spread of $T_{C}, \Delta T_{C}$, and a characteristic size $R_{0}$ of the areas with different $T_{C}$.

Since at the Curie temperature long-range order appears, it is, by definition, a macroscopic characteristic of the material. Then areas of the scale $R_{0}$, mentioned above and attributed to the local $T_{C}$, have to be essentially mesoscopic objects. To be such, the size $R_{0}$ should be much greater than the size $R_{c}$ of the critical fluctuations. Here the concept of the critical fluctuations with their correlation length $R_{c}$ stems from the scaling theory of the critical phenomena. Indeed, the scaling theory of the second order phase transition implies the only scale length which determines the properties of the material near the phase transition. This is the correlation length of the critical fluctuations of the order parameter $R_{c}$ $=a \tau^{-\nu}$, where $\tau=\left(T-T_{C}\right) / T_{C}$ is the reduced temperature and $\nu=2 / 3$ is a critical index in pure materials.

Starting from this basic supposition $\left(R_{0} \gg R_{c}\right)$ Ginzburg $^{5}$ suggested that some number of magnetic clusters appears above $\left\langle T_{C}\right\rangle$ as a result of $T_{C}$ variations. As there is no connection between them, there are randomly oriented magnetized regions, so the mean magnetization equals zero. As the temperature decreases, the connection between the magnetic clusters enhances. Hence a nonzero value of the mean mag- 


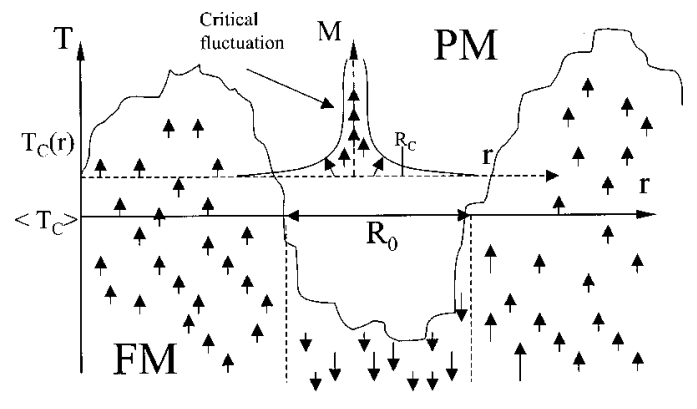

FIG. 1. The local critical temperature $T_{C}(r)$ is randomly distributed around $\left\langle T_{C}\right\rangle$ over the sample in a "percolative" scenario when $R_{0} \gg R_{c}$.

netization appears at temperature $T_{p}$. This temperature $T_{p}$ corresponds to the (geometric) percolation threshold, i.e., the number and size of the ferromagnetic clusters become big enough to join all regions with nonzero local magnetization. This temperature $T_{p}$ can be considered as the true Curie temperature. This is the percolative scenario of the phase transition in the disordered system. This concept received recently extensive development in the work of Korzhenevskii et al. ${ }^{6,7}$ where an explanation of the two scale length phenomenon was also given (see Refs. 1-4, and references therein). The authors discuss the phase transition in an Ising model with large scale disorder. Following the terminology of Refs. 5-7, we assume that due to compositional disorder in the sample we have a local critical temperature $T_{C}(r)$ which varies randomly as a function of position within the sample (Fig. 1). In analogy to scaling theory we introduce the local reduced temperature expressed as $\tau(r)=\left[T-T_{C}(r)\right] /\left\langle T_{C}\right\rangle=\langle\tau\rangle$ $+\Delta \tau(r)$, where $\langle\tau\rangle=\left[T-\left\langle T_{C}(r)\right\rangle\right] /\left\langle T_{C}\right\rangle$ is the average reduced temperature and $\Delta \tau(r)$ is the value of a random variation of $\tau(r)$. The variable $\Delta \tau(r)$ is assumed to be Gaussian with average 0 and a full width at half maximum (FWHM) $=\tau_{1}$. Its spatial dependence is characterized by a correlation function $G\left(r / R_{0}\right)$ defined by

$$
\langle\Delta \tau(0) \Delta \tau(r)\rangle=\left\langle\tau(0) \tau(r)-\langle\tau(r)\rangle^{2}\right\rangle=\tau_{1}^{2} G\left(r / R_{0}\right),
$$

where $G\left(r / R_{0}\right)$ has for limits: $G(0) \rightarrow 1$ and $G(\infty) \rightarrow 0$.

Thus, the disorder is characterized by two parameters: the depth of the $T_{C}$ variations expressed as $\tau_{1}^{2}$ $=\left\langle\left(\Delta T_{C}\right)^{2}\right\rangle /\left\langle T_{C}\right\rangle^{2}$, and the large, but finite correlation lengthscale $R_{0}$ of the disorder. In the scaling theory the main properties of the system are described by value of the reduced temperature $\tau$. The correlation length of the critical fluctuations $R_{c}$ is also function of $\tau$. In order to get the unified formalism, the disorder parameter $R_{0}$ may also be defined using reduced temperature scale via $\tau_{2}=\left(a / R_{0}\right)^{3 / 2}$, where $a$ is a lattice constant. ${ }^{6,7}$ The parameter $\tau_{2}$ corresponds to the point on the temperature scale, where the thermal correlation length reaches the value $R_{c}\left(\tau_{2}\right)=a \tau_{2}^{-\nu}=R_{0}$, with $\nu=2 / 3$. Therefore, the value of ratio $\tau_{1}$ and $\tau_{2}$ defines the scenario of the transition. When $\tau_{2} \gg \tau_{1}$, i.e., $R_{0} \ll a \tau_{1}^{-2 / 3}$, at the temperature point $\tau_{1}$, where the disorder starts to play an essential role, the thermal correlation length $R_{c}=a \tau^{-2 / 3}$ turns to be much larger than the disorder correlation length $R_{0}$. Therefore the disorder is insufficient as in the case of point defects and we get the "homogeneous" scenario. In opposite case $\tau_{2} \ll \tau_{1}, R_{0} \gg a \tau_{1}^{-2 / 3}$, the thermal fluctuations do not destroy the locally ordered regions and that is a basis of a percolative transition (Fig. 1). (For more details see Refs. 5-7). The critical fluctuations do not play a big role in this scenario. They are averaged over $T_{c}$ spread, so that their average correlation length $\left\langle R_{c}\right\rangle$ is of order of $a \tau_{1}^{-\nu}$ through the whole range of the critical temperatures.

Note, however, that the effect of the dipole interaction in the ferromagnets is ignored in the percolative scenario proposed in Refs. 5-7. The dipole interaction blocks actually the percolation. As soon as it happens, a domain structure is formed in the sample. Thus the correlation length of the inhomogeneities does not become infinite at some temperature. The applied magnetic field moves out the domain walls and let the magnetization "percolate" along the field direction. According to Refs. 5-7, the percolation temperature is considered to be $T_{p}=\left\langle T_{c}\right\rangle\left(1+\tau_{1}\right)$. However, the magnetic structure caused by $T_{c}$ variations still remains inhomogeneous. We suggest the continuous growth of the size of the inhomogeneities with lowering temperature and discuss our suggestion below (Sec. IV).

Due to the linear dependence between $T_{C}(r)$ and $\Delta \tau(r)$ and from the assumption of a Gaussian distribution of $\Delta \tau$ with temperature, one can derive the distribution function of $T_{C}$ variations $\rho\left(T_{C}\right)$ with center $\left\langle T_{C}(r)\right\rangle$ and spread $\Delta T_{C}$ $=\tau_{1}\left\langle T_{C}\right\rangle$. For a pure ferromagnet it looks similar to a $\delta$ function of temperature $\rho\left(T_{C}\right)=\delta\left(T-T_{C}\right)$. In case of a disordered system let us consider it to be described by the Gauss law

$$
\rho\left(T_{C}\right)=\frac{1}{\sqrt{2 \pi\left(\Delta T_{C}\right)^{2}}} \exp \left(-\frac{\left(T_{C}-\left\langle T_{C}\right\rangle\right)^{2}}{2 \Delta T_{C}^{2}}\right) .
$$

In the interpretation of our experimental data we show that for invar FeNi alloy the case, when $\tau_{2} \ll \tau_{1}$, is realized and then the ferromagnetic phase transition goes in accordance with the "percolative" scenario. Our experimental findings demonstrating coexistence of two scale lengths of the magnetic correlation ${ }^{3,4}$ are well described by the theoretical concept proposed in Refs. 5-7. Continuing the study of the "two scale" problem, we use the three-dimensional (3D) neutron depolarization analysis as an appropriate method. The magnetization and depolarization of the neutron beam was measured as a function of temperature at different magnetic fields. We fitted the temperature dependence of the magnetization by the convolution of the distribution function $\rho\left(T_{C}\right)$ and the scaling law for the magnetization in a pure system. The parameters of the distribution functions $\left\langle T_{C}\right\rangle$ and $\Delta T_{C}$, were obtained. The analogous procedure being carried out with ND data results in finding the scale parameter of the disorder $R_{0}$ in Eq. (1). The temperature dependence of the characteristic size of the magnetic inhomogeneities was also derived from the data. The result presented in this paper is an extension of the previous works. ${ }^{3,4}$ In the previous works the size of the magnetic inhomogeneities near $T_{C}$ was estimated of order $10^{3}-10^{5} \AA$. In the present study it is transformed to the characteristic size $R_{0}$ of the $T_{C}$ 


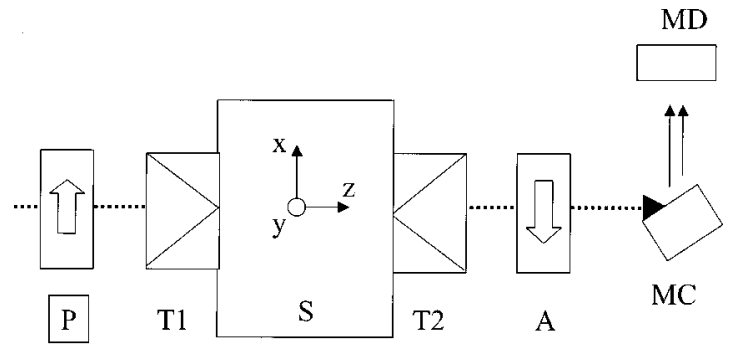

FIG. 2. Scheme of the neutron depolarization setup SP at IRI Delft: $P$ polarizer, $T 1, T 2$ polarization rotators, $S$ sample position, $A$ analyzer, MC monochromator crystals, MD detectors for monochromatic beams.

variations which is equal to $10^{4} \AA$. Therefore the results obtained earlier and those presented here are qualitatively the same. The essential difference is that at present we have applied the concept explaining appearance of the large scale magnetic inhomogeneities above $T_{C}$ and have obtained the quantities $\left\langle T_{C}\right\rangle, \Delta T_{C}$, and $R_{0}$ describing this magnetic phase transition in the disordered system.

The paper is organized in the following way. Section II gives experimental details concerning the sample studied, the setup and the formula describing a ND experiment. The results of the measurement are given in Sec. III. To interpret our data, we use the theoretical concept of the $T_{C}$ variations described above. Section IV presents the interpretation of the experimental results according to this model and the final conclusion.

\section{EXPERIMENTAL}

\section{A. Sample}

The alloy $\mathrm{Fe}_{70} \mathrm{Ni}_{30}$ doped by $0.7 \%$ at. carbon, having fully fcc structure, was chosen for the study. A plate with dimensions $30 \times 10 \times 1.1 \mathrm{~mm}^{3}$ was used as a sample. The addition of carbon is necessary to get fcc structure throughout sample at room temperature. The identification of fcc structure was carried out by means of neutron diffraction on the MiniSFINKS high-resolution Fourier time of flight (TOF) powder diffractometer at the WWR-M reactor, Gatchina. ${ }^{8}$ It is well known also that introduction of a small amount $(\sim 1 \%)$ of carbon in the FeNi alloy results in a growth of its Curie temperature due to an increase of the internal pressure and due to the strong magnetovolume coupling. ${ }^{9}$ The paramagnetic-ferromagnetic phase transition is smeared out between 390-400 K. The transition point determined by SANS measurements is equal to $T_{f}=386 \pm 1 \mathrm{~K}$ ( the index $f$ is referred to ferromagnet). It is the temperature which the correlation radius of critical fluctuations becomes maximal and saturated at.

\section{B. Neutron depolarization: experimental}

The neutron depolarization measurements described in this paper were carried out using the mirror setup SP at IRI, Delft. Figure 2 gives its schematic outline. A polychromatic neutron beam emerging from a 2 MW swimming-pool type reactor is polarized by a mirror polarizer $(P)$. Using rotator
(T1) the polarization of the beam can be successively rotated towards one of the laboratory axes $j(j=x, y, z)$. After the transmission through the sample holder containing the sample, positioned in the sample box of $\mu$ metal $(S)$ the polarization can be successively analyzed along the directions $i(i=x, y, z)$, using a second polarization rotator (T2) and an analyzer $(A)$. By Bragg reflection at a monochromator crystal (MC), neutrons with wavelength $\lambda=(0.32$ $\pm 0.02) \mathrm{nm}$ are reflected into the neutron detector (MD). A magnetic field is applied to the sample in the y direction. It is created by a coil wound on the sample. In order to minimize the sample demagnetization field, the sample together with the coil is positioned in a magnetic yoke which short-circuits any flux emerging from it. In turn the yoke is mounted in a furnace whose temperature could be regulated to better than $0.1 \mathrm{~K}$ between 300 and $500 \mathrm{~K}$.

The relation between the polarization of the neutron beam $P_{i j}$ and the intensity $I_{i j}$ measured in the detector (MD) is given by

$$
P_{i j}=\delta_{i j}-\left(I_{i j} / I_{\text {shim }}\right),
$$

where $I_{\text {shim }}$ is the intensity of the fully depolarized beam. The first and second subscripts denote the projections of the initial and transmitted polarization on the $i, j$ axes, respectively. All measured elements are normalized to correct for the depolarization caused by the setup. This normalization factor $P_{0}$ is determined from the measurement of the length of the polarization vector well above $\left\langle T_{C}\right\rangle$.

\section{Neutron depolarization: theory}

The polarization $\vec{P}^{\prime}$ of the beam after transmission is related to the initial polarization $\vec{P}^{0}$ by the $3 \times 3$ depolarization matrix $\hat{D}$ :

$$
\vec{P}^{\prime}=\hat{D} \cdot \vec{P}^{0} .
$$

In general, this change involves both a rotation of the polarization vector, due to the magnetic induction in the sample $\vec{B}(\vec{r})$, and a change of its length, i.e., a decrease of the degree of polarization, due to inhomogeneities in the magnetic induction within the sample $\Delta \vec{B}(\vec{r})=\vec{B}(\vec{r})-\langle\vec{B}\rangle$, where $\vec{B}(\vec{r})$ is local magnetic induction.

When no net rotation of polarization occurs $(\langle\vec{B}\rangle=0)$, the measured depolarization matrix $\hat{D}$ is diagonal. The relation between the elements of $\hat{D}$ and the micromagnetic state of the isotropically disordered media can be given through the integral cross section of neutron scattering $\sigma_{\Psi}$ within the detector acceptance angle $\Psi$ by the expression ${ }^{10,11}$

$$
D_{x x}=D_{y y}=\exp \left[-(3 / 2) \sigma_{\Psi} L\right], \quad D_{z z}=\exp \left(-\sigma_{\Psi} L\right),
$$

where $L$ is the sample thickness and the neutron velocity $v$ is parallel to the $z$ axis (Fig. 2). The neutron magnetic scattering cross section $\sigma_{\Psi}$ is determined by the correlation function of the magnetization density along the neutron trajectory. It can be written as ${ }^{10,11}$ 


$$
\begin{gathered}
\sigma_{\Psi}=\frac{1}{3}\left(\frac{2 \mu_{n} B(T)}{\hbar v}\right)^{2} R_{d}, \\
R_{d}=\frac{1}{2} \int_{-\infty}^{\infty} d z\left\langle\vec{m}(x, y, z) \vec{m}\left(x, y, z^{\prime}\right)\right\rangle,
\end{gathered}
$$

where $\vec{m}(x, y, z)$ is an unit vector along the magnetization and $\mu_{n}$ is the neutron moment. For convenience we introduce the wavelength dependent constant $c=\left(2 \mu_{n} / \hbar v\right)^{2}$ $=2.14 \times 10^{29} \lambda^{2}\left[m^{-4} T^{-2}\right]$ that will be used in the expressions. It is readily seen from Eq. (6) that the cross section $\sigma_{\Psi}$ depends on the size as well as the shape of the magnetic correlation $\left\langle\vec{m}(x, y, z) \vec{m}\left(x, y, z^{\prime}\right)\right\rangle$. As proved in Refs. 10,11 (see also Refs. 3,4), in the case of critical fluctuations the intrinsic induction is large at the center of the fluctuation only and falls off drastically as $1 / r$ towards the periphery giving a small value of the parameter $R_{d}$. Therefore, the cross section $\sigma_{\Psi}$ should be small for any reasonable correlation radius of the critical fluctuations (up to $10^{5} \AA$ ). A small value of $\sigma_{\Psi}$ leads to absence of any measurable change of the polarization. Then Eq. (5) is simplified to $D_{i i}$ $\approx 1,(i=x, y, z)$ and Eq. (4) to $\vec{P}^{\prime} \approx \vec{P}^{0}$. So a measurable depolarization can be attributed exclusively to the appearance of the magnetic clusters with relatively homogeneous distribution of the induction. This conclusion is important for the ND study of the magnetic structure near $T_{C}$ since the critical fluctuations can be ignored in the interpretation of the ND data.

Consider the case when an external magnetic field $\vec{H}$ is applied along the $y$ axis, so that the sample is magnetized along the field $\langle\vec{B}\rangle \| \vec{H}$. Then the matrix $\hat{D}$ can be written ${ }^{12-14}$

$$
\hat{D}=\left(\begin{array}{ccc}
D_{\perp} \cos (\phi) & 0 & D_{\perp} \sin (\phi) \\
0 & D_{\|} & 0 \\
-D_{\perp} \sin (\phi) & 0 & D_{\perp} \cos (\phi)
\end{array}\right)
$$

where

$$
\phi=\sqrt{c}\langle\vec{B}\rangle L
$$

is the rotation angle of the precession of the polarization vector in the mean magnetic induction $\langle\vec{B}\rangle$ over the sample length $L$. The quantities $D_{\|}$and $D_{\perp}$ are dissipative coefficients of the parallel and perpendicular (to the field) components of the polarization vector. In full analogy to Eqs. (5),(6), they are related with the local variation of the magnetic induction $\Delta B$ and its correlation length $R_{d}$ by the expression $^{12,13}$

$$
D_{\|, \perp}=\exp \left(-\frac{1}{2} c\left\langle\Delta B^{2} R_{d}\right\rangle L\left(1-\gamma_{\|, \perp}\right)\right),
$$

where $\gamma_{\|, \perp}$ are related with the mean square cosines of the local magnetic induction and with the intrinsic anisotropy of the ND. The product $\left\langle\Delta B^{2} R_{d}\right\rangle$ is an average over the sample volume.
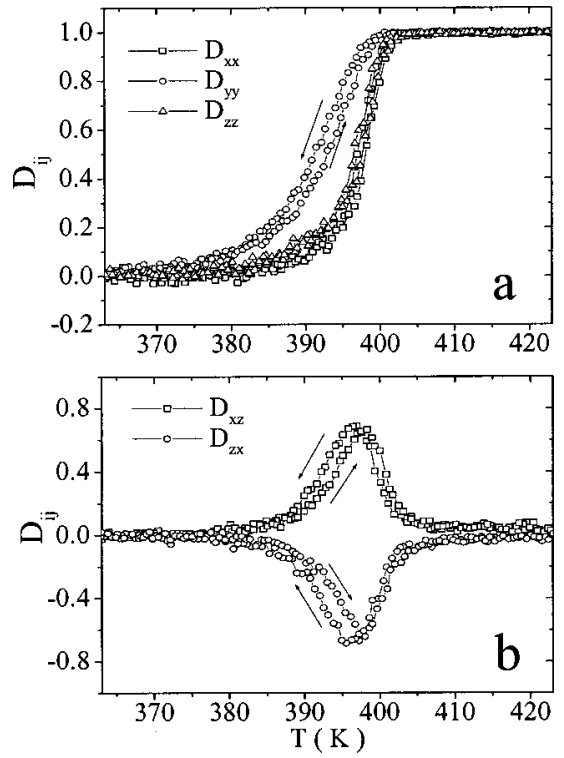

FIG. 3. Temperature dependence of the diagonal $D_{x x}, D_{y y}, D_{z z}$ (a) and nondiagonal $D_{x z}, D_{z x}$ (b) elements of the depolarization matrix at $H=0.05 \mathrm{G}$.

The quantities $D_{\|, \perp}$ are calculated from the measured elements of the depolarization matrix [Eq. (7)] by the following combinations of its elements:

$$
D_{\|}=D_{y y} \quad D_{\perp}=\left(D_{x x} \cdot D_{z z}-D_{x z} \cdot D_{z x}\right)^{1 / 2} .
$$

According to Eq. (7) the rotation angle $\phi$ is derived from the matrix $\hat{D}$ :

$$
\phi=\tan ^{-1}\left(\frac{D_{x z}-D_{z x}}{D_{x x}+D_{z z}}\right)
$$

\section{RESULTS}

\section{A. Three-dimensional depolarization measurement}

The elements of the matrix $\hat{D}$ were measured from far below $T_{C}$ up to far above $T_{C}$, consecutively, in various magnetic fields $H_{y}=0.05,1.5,3,10 \mathrm{G}$. The raw depolarization data are presented in Figs. 3,4. Figure 3 gives the temperature dependence of the elements of the depolarization matrix $D_{x x}, D_{y y}, D_{z z}$ (diagonal) and $D_{x z}, D_{z x}$ (nondiagonal) at the field $H=0.05$ G. The nondiagonal elements shown are antisymmetrical: $D_{x z}=-D_{z x}$ [Fig. 3(b)]. In the case of applied field for the diagonal and nondiagonal elements the data are presented in Figs. 4(a) and 4(b), respectively. The element $D_{y y}$ of the matrix, characterizing the depolarization along the applied field, decreases while the perpendicular elements $D_{x x}, D_{z z}$ show an oscillatory behavior with diminishing temperature. The nondiagonal elements are antisymmetrical $\left(D_{x z}=-D_{z x}\right)$ and are also oscillating with temperature. The other elements of the depolarization matrix are zero within the experimental error both for $H=0.05 \mathrm{G}$ and for $H$ $=10$ G. Two factors contribute to the error of the measurement, the statistics of the measurement and uncontrolled deviation of the magnetic field direction from the $y$ axis which 

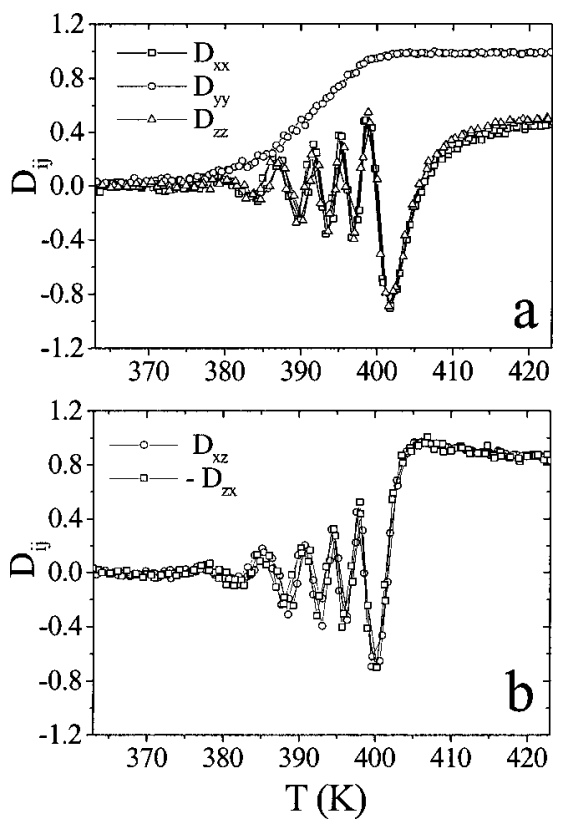

FIG. 4. Temperature dependence of the diagonal $D_{x x}, D_{y y}, D_{z z}$ (a) and nondiagonal $D_{x z}, D_{z x}$ (b) elements of the depolarization matrix at $H=10.0 \mathrm{G}$.

is evaluated of order of $2^{\circ}-3^{\circ}$. The former factor does not exceed $0.5 \%$, while the latter gives a main contribution of order of $3-5 \%$.

From Figs. 3,4 one can conclude the following. First, only two nondiagonal elements $D_{x z}$ and $D_{z x}$ are antisymmetrical and oscillating as functions of temperature. This implies the existence of a mean induction in the sample along the $y$ direction, while the other directions are magnetically nondistinguished. Therefore the experimental results can be treated by Eqs. (7) $-(11)$.

From the raw data the values $D_{\|, \perp}$ and $\phi$ were calculated according to Eqs. (10),(11). The temperature dependence of the quantities $D_{\|}$and $D_{\perp}$ for both small (0.05 G) and large $(10 \mathrm{G})$ magnetic field are given in Figs. 5 and in 6, respectively. At $T \gg T_{C}$ the polarization of the transmitted beam is equal to the incident polarization $\vec{P}_{0}$. As the temperature decreases to the transition temperature $T_{0} \approx 405$, depolarization

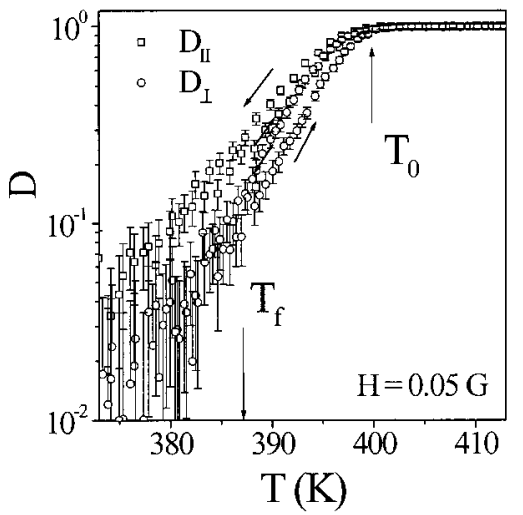

FIG. 5. Temperature dependence of the quantities $D_{\|}$and $D_{\perp}$ at $H=0.05 \mathrm{G}$.

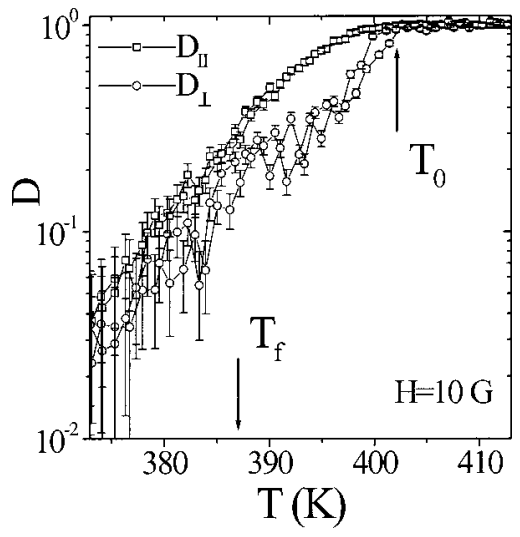

FIG. 6. Temperature dependence of the quantities $D_{\|}$and $D_{\perp}$ at $H=10 \mathrm{G}$.

appears. It increases over a wide temperature range of some tens of degrees. According to Eq. (9) the neutron depolarization is attributed to the appearance of magnetic inhomogeneities with $B \approx 10^{2}-10^{3} \mathrm{G}$ and size about $10^{3}-10^{5} \AA$. Temperature hysteresis of $D_{\|, \perp}$ is observed at the field $H$ $=0.05 \mathrm{G}$. The sequence of the measurements is shown in the figures by the arrows. The hysteresis loop is suppressed at the field $H=10 \mathrm{G}$. Note also that the value $D_{\perp}(H$ $=10 \mathrm{G})$ is a few times lower than $D_{\perp}(H=0.05 \mathrm{G})$ in the range $\left[T_{C}, T_{0}\right]$, while the value $D_{\|}(H=10 \mathrm{G})$ has a hardly remarkable change. The explanation of this fact is presented below.

The rotation angle $\phi$ is given in Fig. 7 as a function of temperature at different magnetic fields $H=0.05,1.5$, $3.0,10 \mathrm{G}$ as the alloy was cooled and heated. According to Eq. (8), it is proportional to the mean magnetic induction $\langle B\rangle$. The rotation angle is corrected for the rotation caused by the external magnetic field by subtracting the rotation angle $\phi_{\min }$, which is measured at temperatures where the sample is paramagnetic. Thus in the paramagnetic range $\phi$ $=0$ and it grows with decreasing temperature at $H=1.5,3.0$, and $10 \mathrm{G}$. The magnitude of $\phi$ is maximal at $H=10 \mathrm{G}$ while at $H=0.05 \mathrm{G}$ it is practically equal to zero in the whole temperature range. From Eq. (8) a value of $\langle B\rangle$ can be obtained. Thus at $H=10 \mathrm{G}$ at $T=393 \mathrm{~K}, \phi$ equals $15 \mathrm{rad}$, $\mathrm{L}=0.11 \mathrm{~cm}$ and thus $\langle B\rangle \approx 930 \mathrm{G}$. As is seen from Fig. 7

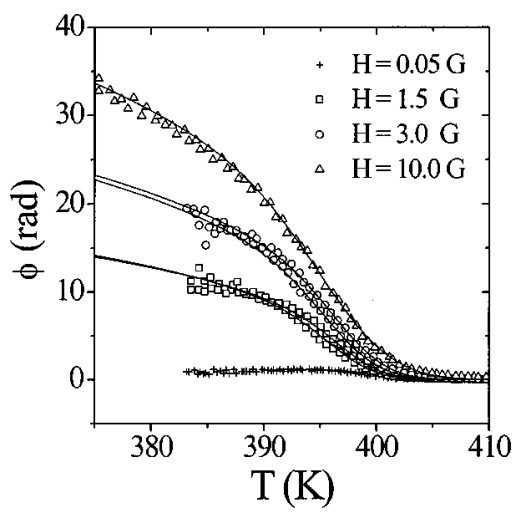

FIG. 7. Temperature dependence of the rotation angle of the polarization vector $\phi$ at different magnetic fields: $0.05,1.5,3,10 \mathrm{G}$. 


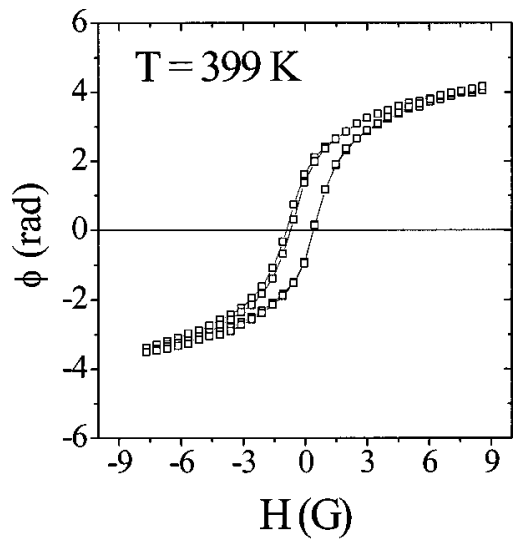

FIG. 8. The magnetic field dependence of the rotation angle of the polarization vector $\phi$ at $T=399 \mathrm{~K}$.

the temperature hysteresis of $\phi$ is also observed at $H=1.5$ and $3.0 \mathrm{G}$. It is suppressed by the magnetic field $H=10 \mathrm{G}$. The temperature dependence of the rotation angle $\phi$ is interpreted using the concept of a probability function $\rho\left(T_{C}\right)$ which describes the variations of the Curie temperatures over the sample. This concept is extensively discussed in the next chapter.

The elements of depolarization matrix were also measured as function of the field in the range $H=[-9,9] \mathrm{G}$ at constant temperature $T=399 \mathrm{~K}$. The magnetic field dependence of the rotation angle $\phi$ of the polarization vector is given in Fig. 8. The magnetic hysteresis in the mean magnetic induction is observed. The width of the hysteresis loop (at $\langle B\rangle=0$ ) is approximately equal to $1.5 \mathrm{G}$. Figure 9 gives the quantities $D_{\|}, D_{\perp}$ as a function of the magnetic field at $T=399 \mathrm{~K}$. The value of $D_{\perp}$ decreases remarkably as the field increases while $D_{\|}$remains almost unchanged. The hysteresis observed in the values $D_{\|, \perp}$ is related to reorganization of the magnetic structure when the magnetic field changes sign and is varied over the range of the small values. The value of the hysteresis in the depolarization of $10 \%$ at zero field (Fig. 9) matches the value of the hysteresis loop at the temperature scan (Fig. 5).

In our opinion, the strong change of the depolarization $D_{\perp}$ together with the weak change of $D_{\|}$, as magnetic field is

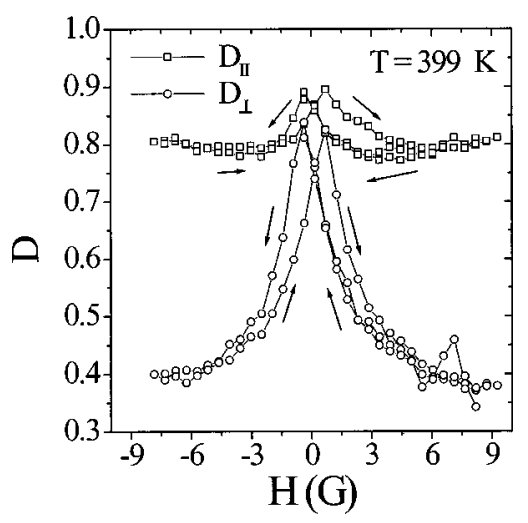

FIG. 9. The magnetic field dependence of the quantities $D_{\|}$and $D_{\perp}$ at $T=399 \mathrm{~K}$.

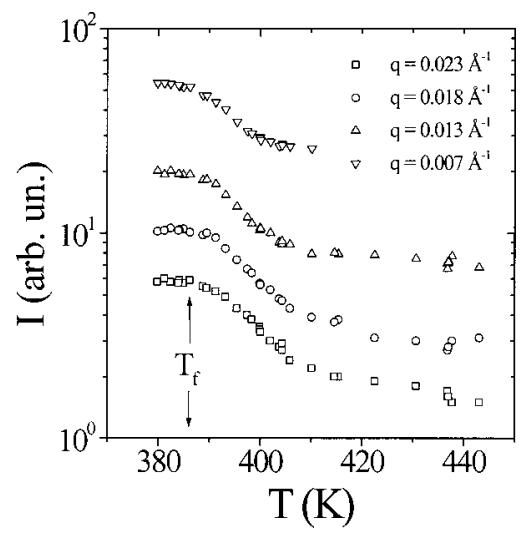

FIG. 10. The small angle scattering intensity versus temperature for different momentum transfers $q$.

applied, demonstrates the enhancement of the extra factor $D_{\text {ext }}$ in depolarization $D_{\perp}$ that is caused by variation of the rotation angle over the beam cross section. ${ }^{12,13}$ Indeed, if $f(\phi)$ is a normalized distribution function of the rotation angle of polarization vector in the magnetic field over the beam cross section, then the depolarization elements perpendicular to the applied field $D_{x x}, D_{x z}, D_{z x}, D_{z z}$ and therefore $D_{\perp}$ will be decreased by the factor

$$
D_{\text {ext }}=\int_{0}^{\infty} f(\phi) \cos (\phi-\langle\phi\rangle) d \phi .
$$

If distribution function $f(\phi)$ is assumed to be Gaussian with squared standard deviation $\sigma_{\phi}^{2}$, then substitution of Gaussian instead of $f(\phi)$ in Eq. (12) yields $D_{\text {ext }}=\exp \left(-\sigma_{\phi}^{2} / 2\right)$. The variation of $\phi$ is possibly caused by a gradient of the temperature over the sample. An estimation shows that the gradient of the temperature over the sample of about $1 \mathrm{~K}$ results in about 1 rad variations of the rotation angle over the beam cross section (Fig. 6) and therefore in the extra depolarization of the perpendicular components $D_{\perp}$ of about $30 \%$.

As can be observed in Fig. 8, by the applied field the magnetic moment of these locally ordered regions tends to align. The magnetic induction $\langle B\rangle$ grows rapidly with $H$ and reaches saturation at a magnetic field of $10 \mathrm{G}$. In comparison, the depolarisation $D_{\|}$in Fig. 9 only shows a weak field dependence. From this we conclude that the applied magnetic field is sufficiently strong to move out the domain walls, but is weak in comparison with the anisotropy field. So, when a magnetic field of $10 \mathrm{G}$ is applied, all magnetic inhomogeneities contribute to the mean magnetic induction through the whole temperature range but the magnetic inhomogeneous structure is hardly effected in this field range.

\section{B. Small-angle neutron scattering measurement}

The SANS experiment was performed on the multidetector small-angle polarized-neutron scattering setup "Vector" at WWR-M reactor in St.Petersburg Nuclear Physics Institute. ${ }^{15}$ The temperature dependence of SANS intensity in the momentum transfer range $3 \times 10^{-3} \leqslant q \leqslant 3$ $\times 10^{-2} \AA^{-1}$ was measured through the transition with a step of $1 \mathrm{~K}$. Figure 10 displays the scattering intensity $I(T)$ 


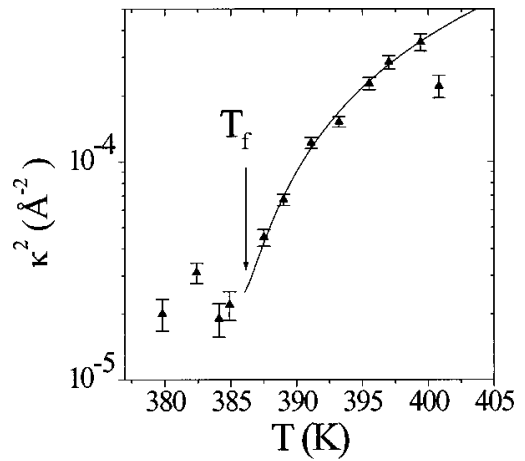

FIG. 11. The temperature dependence of square of inverse correlation radius $R_{c}^{-2}(T)$. The calculated dependence with the parameters obtained from the fits is plotted by solid line.

for different values of momentum transfer $q$ in the temperature interval 380-450 K. The SANS intensity $I(T)$ does not change in the paramagnetic temperature range. With decreasing temperature, the intensity $I(T)$ increases, demonstrating the appearance of critical fluctuations in the sample. The intensity reaches its saturation at $T \approx 385 \mathrm{~K}$.

The SANS intensity as a function of the momentum transfer $q$ is well described by Lorentzian

$$
I(q)=A\left(q^{2}+\kappa^{2}\right)^{-1},
$$

where the $A$ and $\kappa$ are the scattering amplitude and the inverse correlation length, respectively. This demonstrates that we deal with conventional critical fluctuations. The parameters $A$ and $\kappa^{2}$ were obtained from a least squares fits of Eq. (13) to the data.

The temperature dependence of the square of inverse correlation radius $\kappa^{2}(T)=R_{c}^{-2}$ is presented in Fig. 11. We see that $\kappa^{2}$ decreases as temperature decreases until a certain temperature $T_{f}$ and then remains almost constant for $T$ $<T_{f}$. From Figs. 10 and 11 is seen that the critical range spreads over a wide temperature band from $T_{0} \approx 410 \mathrm{~K}$, where critical scattering starts to increase, to $T_{f} \approx 386 \mathrm{~K}$, where it is saturated. The magnetic system is paramagnetic at $T>T_{0}$ and it has a hard domain structure at $T<T_{f}\left(T_{f}\right.$ is referred to ferromagnet). We set the temperature $T_{f}$, where the inverse correlation length $\kappa$ becomes constant, as the transition temperature of the alloy. The temperature dependence of $\kappa^{2}$ is described satisfactory by the power law $\kappa^{2}(\tau)=\kappa_{1}^{2}+\kappa_{0}^{2} \cdot \tau^{2 \nu}$ with $\left[\tau=\left(T-T_{f}\right) / T_{f}\right], \kappa_{1}^{2}=\left(R_{c}^{\max }\right)^{-2}$, $\kappa_{0}^{2}=\left(R_{c}^{\mathrm{min}}\right)^{-2}, \nu \approx 2 / 3$ typical for ferromagnets. The line in Fig. 10 represents this expression with $R_{c}^{\max }=200 \pm 20 \AA$, $R_{c}^{\min }=6 \pm 2 \AA$, and $T_{f}=386 \pm 1 \mathrm{~K}$.

The parameter $\kappa_{0}$, being a coefficient in the power law relation $R_{c} \approx \kappa_{0}^{-1} \tau^{-\nu}$, is effectively the inverse correlation radius at $\tau \sim 1$. The value $\kappa_{0}^{-1}=6 \AA$ indicates the presence of spin fluctuations of this scale in the far paramagnetic region. The spin correlations of this size (about $6-8 \AA$ ) in the invar FeNi alloys well above $T_{C}$ were reported in a number of papers. ${ }^{16-18}$ This gives an additional confidence to the fitting procedure performed with the power law relation of $R_{c}(\tau)$. The presence of these spin fluctuations well above $T_{C}$ is explained by a sensitive dependence of the magnetic mo- ment on the atomic volume or, by a moment-volume instability resulting also in Invar effect.

Another peculiarity is the fact that the inverse correlation length $\kappa$ does not appear to go to zero. This behavior is commonly observed in disordered systems ${ }^{19,20}$ and in these cases is treated to originate from the spread on the transition temperature. With use of the power law relation one can estimate the $\tau$ range which $R_{c}(\tau)$ is averaged at $R_{c}^{\max }$ $\sim \kappa_{0}^{-1} \tau_{1}^{-\nu}$. This estimation gives the value of $\tau_{1}$ about 0.005 , but with the low accuracy of about $30 \%$. Finally it is clear that the correlation length of the critical fluctuations is not assumed to be unique and is averaged over the $T_{C}$ variations.

\section{DISCUSSION}

In order to interpret the above data we summarize the conclusions which follow from the SANS and depolarization experiments. The complementary analysis shows a coexistence of two types of the correlations in the system in the temperature range $T_{0}>T>T_{f}$. First, there are the usual critical fluctuations above $T_{f}$ which cannot produce strong neutron depolarization (see Sec. II). Their correlation length $R_{c}$ $=\kappa^{-1}$ was obtained from SANS data and it is about $100-$ $200 \AA$ over the whole range. Secondly, the large depolarization above $T_{f}$ is attributed to the existence of large magnetic inhomogeneities in the system. The size and/or the number of these inhomogeneities rises as temperature decreases. The presence of these inhomogeneities determines the properties of the magnetic system in the temperature range $T_{f}<T$ $<T_{0}$ which is considered as a critical range. Hence, the magnetic state of the sample in this critical temperature range looks similar to ferromagnetic drops in a paramagnetic matrix with critical fluctuations both in the ferromagnetic and paramagnetic regions. These locally ordered magnetic regions grow in the paramagnetic phase and form a large percolative cluster. The correlation length of the ferromagnetic cluster exceeds the correlation length of the critical fluctuations $R_{c}$ at least by a factor of 10 . In this case the dominating lengthscale is not the correlation length of thermal fluctuations but the characteristic length of the ordered regions. Therefore this transition goes in accordance with the "percolative" scenario. With lowering temperature this system of the ferromagnetic regions is smoothly transformed into the domain structure caused by the dipole interaction. Thus in this study we follow changes of the characteristic size of the magnetic inhomogeneities formed at $T \sim T_{C}$ and turning into the domain size at $T \ll T_{C}$. The directions of magnetization of these inhomogeneities are random, so that the average magnetization is equal to zero both above $\left\langle T_{C}\right\rangle$ and below it. However, the magnetic moments of these locally ordered regions may be aligned by the magnetic field. Then a mean magnetic induction (about $10^{2}-10^{3} \mathrm{G}$ ) appears as a response to the external magnetic field (about 1-10 G). The mean magnetic induction is composed of the magnetic moments of the locally ordered regions over the whole sample volume.

The data of the depolarization, the rotation angle $\phi$ and SANS give an evidence of the smeared phase transition: the 
Curie temperature fluctuates over the sample around the average $\left\langle T_{C}\right\rangle$. In Ref. 9 two main reasons of variations of the Curie temperature and smearing the transition are pointed out. One of them is an enhancement of some component concentrations over the sample volume when the phase diagram is complex due to a competition of exchange interactions. Another one is the inhomogeneous mechanical deformation leading to local distortions of the lattice in the case of the strong magnetostriction. It is experimentally shown ${ }^{9}$ that both factors are equally presented in Invar FeNi alloys. Rough estimations demonstrate that large scale variations of the component concentrations $\Delta c$ of about $0.1 \%$ result in fluctuations of the Curie temperature in the alloy $\left\langle\Delta T_{C}^{2}\right\rangle^{1 / 2}$ $\sim 1-3 \mathrm{~K}$. It can be estimated that volume variations $\Delta V$ $\sim 0.1 \%$ lead to $\left\langle\Delta T_{C}^{2}\right\rangle^{1 / 2} \sim 10-30 \mathrm{~K}$. There are no experimental proofs that the latter reason of $T_{C}$ variations is the dominant one, however, it is beyond doubt that it cannot be neglected. The latter reason is essentially important for the Invar $\mathrm{FeNi}$ alloy doped with carbon which tends to form clusters and then creates regions of different volume density.

\section{A. Temperature dependence of the mean magnetization}

We assume above that there are local $T_{C}$ variations and magnetically ordered regions appear above $\left\langle T_{C}\right\rangle$. We suppose also that the magnetic induction in the locally ordered region is described by the scaling expression

$$
B(\tau)=B_{0}|\tau|^{\beta},
$$

where $\tau=\left(T-T_{C}\right) / T_{C}$ is the local reduced temperature, $\beta$ $\approx 0.37$ is the critical index of magnetization of the pure ferromagnet. As a result the mean magnetic induction of the sample can be expressed by the convolution $B\left(T, T_{C}\right)$ [Eq. (14)] and $\rho\left(T_{C}\right)$ [Eq. (2)]:

$$
\langle B(T)\rangle=B_{0} \int_{T}^{\infty} \rho\left(T_{C}^{\prime}\right)\left|\frac{T-T_{C}^{\prime}}{T_{C}^{\prime}}\right|^{\beta} d T_{C}^{\prime},
$$

where $B_{0}$ is a fitting parameter which takes into account the demagnetization from magnetic interaction between ordered regions. Upon substituting this expression into the expression (8) $\phi=\sqrt{c}\langle B\rangle L$ relating the rotation angle of polarization vector $\phi$ and the mean magnetic induction $\langle B\rangle$, we obtain

$$
\phi=\sqrt{c} B_{0} \int_{T}^{\infty} \rho\left(T_{C}^{\prime}\right)\left|\frac{T-T_{C}^{\prime}}{T_{C}^{\prime}}\right|^{\beta} L d T_{C}^{\prime} .
$$

Substituting for $\rho\left(T_{C}\right)$ the Gaussian [Eq. (2)] in Eq. (16), the best fit procedure for the experimental data was carried out. As a result, the three parameters $B_{0}, \Delta T_{C}$ and $\left\langle T_{C}\right\rangle$ were found.

Figure 12 shows the experimentally measured points of the rotation angle (closed squares) and the theoretical curve calculated from Eq. (16) (solid line) with the Gaussian [Eq. (2)] as the probability function $\rho\left(T_{C}\right)$ in the magnetic field of $10 \mathrm{G}$. The parameters of the fit at different magnetic fields and temperature regimes (cooling and heating) are given in Table I. The fitted curves are plotted in Fig. 7 as solid lines.

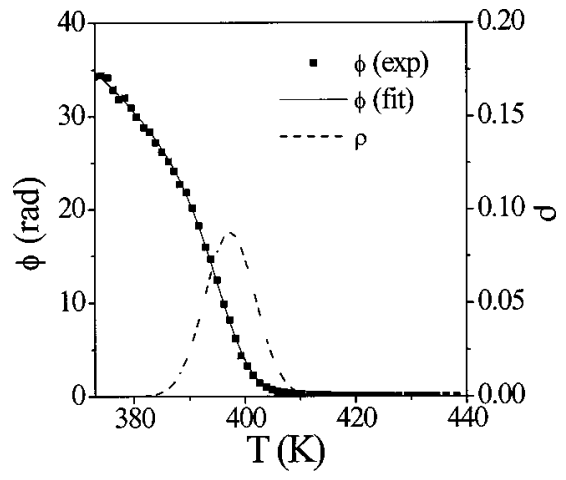

FIG. 12. Temperature dependence of the rotation angle of the polarization vector $\phi(H=10 \mathrm{G})$ [squares: experimental points; solid line: theoretical calculation Eq. (16)] and the probability function $\rho(T)$ described by a Gauss law with fitting parameters $\left\langle T_{C}\right\rangle$ $=397.2 \mathrm{~K}$ and $\Delta T_{C}=4.55 \mathrm{~K}$.

It is seen from Table I that for different conditions of experiments (cooling-heating, magnetic field) we find $\left\langle T_{C}\right\rangle=397$ \pm 1 , independent of the applied field, while $\Delta T_{C}$ grows slightly with applied magnetic field. This may be connected with the alignment of the domain inside inhomogeneities into the field direction. Since at $H=10 \mathrm{G}$ more inhomogeneities contribute to the mean magnetic induction, $\Delta T_{C}$ $=4.55 \mathrm{~K}$ obtained at this field may be considered as the most correct value. Furthermore, an increase of the paramagnetic magnetization in the critical region with applied field is neglected. Note that according to Refs. 5-7, the percolation temperature is approximately equal to $T_{p} \approx\left\langle T_{C}\right\rangle+\Delta T_{C}$ $=401.5 \mathrm{~K}$.

A large difference exists between $T_{f}=386 \mathrm{~K}$ obtained from SANS and $\langle T\rangle=397 \mathrm{~K}$ from the fit above. The difference between the sensors in the SANS and ND setups does not exceed $2-3 \mathrm{~K}$, so this does not explain the observed discrepancy. The discrepancy is explained as follows. The temperature $T_{f}$ obtained by SANS is actually defined as a boundary between the ferromagnetic state with a "hard" do-

TABLE I. The LSM fitting parameters of the probability function $\rho\left(T_{C}\right)$ from the rotation angle data.

\begin{tabular}{lccc}
\hline \hline $\begin{array}{l}\text { Magnetic and } \\
\text { temperature } \\
\text { conditions }\end{array}$ & $B_{0}(\mathrm{G})$ & $\left\langle T_{C}\right\rangle(\mathrm{K})$ & $\Delta T_{C}(\mathrm{~K})$ \\
\hline $\begin{array}{l}H=10 \mathrm{G}, \\
\text { cooling }\end{array}$ & $4620 \pm 5$ & $397.2 \pm 0.1$ & $4.55 \pm 0.05$ \\
\hline $\begin{array}{l}H=3 \mathrm{G}, \\
\text { cooling }\end{array}$ & $3120 \pm 5$ & $397.0 \pm 0.1$ & $3.47 \pm 0.05$ \\
\hline $\begin{array}{l}H=3 \mathrm{G}, \\
\text { heating }\end{array}$ & $3150 \pm 5$ & $397.8 \pm 0.1$ & $3.35 \pm 0.05$ \\
\hline $\begin{array}{l}H=1.5 \mathrm{G}, \\
\text { cooling }\end{array}$ & $1940 \pm 5$ & $397.1 \pm 0.1$ & $3.00 \pm 0.05$ \\
\hline $\begin{array}{l}H=1.5 \mathrm{G}, \\
\text { heating }\end{array}$ & $1900 \pm 5$ & $397.9 \pm 0.1$ & $2.90 \pm 0.05$ \\
\hline \hline
\end{tabular}


main structure and the critical region where critical fluctuations and large ferromagnetic inhomogeneities coexist. Further the center $\left\langle T_{C}\right\rangle$ of the probability function $\rho\left(T_{C}\right)$ obtained from ND shows the middle point of the critical region. Thus, the temperatures $T_{f}$ and $\left\langle T_{C}\right\rangle$ characterize the low boundary and the center of the magnetic phase transition in such a disordered system, respectively. This confirms that the critical fluctuations play a secondary role of at this transition. First, their average length $R_{c}$ is much less than the size of the magnetic inhomogeneities. Second, the magnetic inhomogeneities appear some degrees above $T_{f}$, where $R_{c}$ becomes maximal and saturated. Finally we can conclude that the critical fluctuations are suppressed by the appearance of large scale magnetic inhomogeneities. In spite of this suppression, the transition in the local regions is supplied by the energy of the critical fluctuations. Therefore the mechanism of the transition in these separated regions is one of the second order.

\section{B. Temperature dependence of the neutron depolarization}

As we see the value $D_{\perp}$ includes the extra factor $D_{\text {ext }}$ [Eq. (12)] under the condition of the nonzero applied field. Therefore using Eq. (9) we cannot obtain the true temperature dependence of $\gamma_{\|} . D_{\perp}$ cannot be also considered in a way anologous to the analysis of temperature dependence of the rotation angle $\phi(T)$. On the other hand, the value $D_{\|}$can be analyzed in the same manner. Fortunately, two circumstances allow us to ignore the temperature dependence of $\gamma_{\|}$in Eq.(9). First, the polarization $D_{\|}$changes little when the magnetic field is applied. From this follows that $\gamma_{\|}$does not change significantly also and the magnetic system is close to disorder. This means that the applied magnetic field moves out the domain walls but the magnetic structure caused by $T_{C}$ variations remains inhomogeneous. Second, as it is shown in Refs. 12,13, $\gamma_{\|}$may change in the range from 0.25 (full disorder) to 0.75 (full alignment). As compared with a total change of the value $\ln D_{\|}$which is from 0.01 up to 10 near the phase transition, a change of $\gamma_{\|}$in the same range can be roughly neglected. We set below the value of $\left\langle\gamma_{\|}\right\rangle$ $=1 / 2$.

In analogy to Eq. (16), the temperature dependence of the neutron depolaization $-\ln D_{\|}$for magnetic field $H=10 \mathrm{G}$ (see Fig. 13) was fitted with the convolution of the product $\left[\Delta B\left(T, T_{C}\right)\right]^{2} R_{d}\left(T, T_{C}\right)$ and $\rho\left(T_{C}\right)$ :

$$
-\ln D_{\|}=\frac{1}{4} c L \int_{T}^{\infty} \rho\left(T_{C}^{\prime}\right) B_{0}^{2}\left|\frac{T-T_{C}^{\prime}}{T_{C}^{\prime}}\right|^{2 \beta} R_{d}\left(T, T_{C}^{\prime}\right) d T_{C}^{\prime} .
$$

To fit the data in Fig. 13, we take again a power-law description with Gaussian distribution of transition temperature $\rho\left(T_{C}\right)$. It is supposed that the local magnetic induction in the magnetic inhomogeneities at $T<T_{C}$ is described by the scaling law Eq. (14) where $\tau$ is the local reduced temperature. The critical index $\beta$ is doubled because of the square of the local magnetic induction in Eq. (9). We assume that the size of the inhomogeneities grows with decreasing local reduced temperature also according to a power law

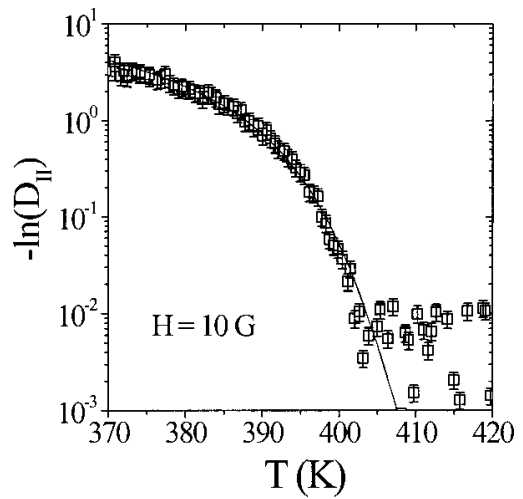

FIG. 13. Temperature dependence of the depolarization $-\ln D_{\|}$ at field $H=10 \mathrm{G}$. The solid line is a curve calculated from Eq. (17) with the Gaussian [Eq. (2)] centered at $\left\langle T_{C}\right\rangle=397 \mathrm{~K}$ with the spread $\Delta T_{C}=4.55 \mathrm{~K}$.

$$
R_{d}\left(T, T_{C}\right)=R_{d}(0)\left(\frac{T_{C}-T}{T_{C}}\right)^{\Delta} .
$$

The assumption Eq. (18) comes in contradiction with the percolative concept proposed in Refs. 5-7. According to Refs. 5-7, the temperature of the transition is determined as that $T$, where an infinite percolative cluster appears. Thus the magnetic correlation length $R_{d}$ must be infinitely large at $T$ $=\left\langle T_{C}\right\rangle+\Delta T_{C}$, while Eq. (18) supposes a continuous growth of $R_{d}$ through the whole critical range. However, the neutron depolarization technique provides information on the average size of inhomogeneities which doesn't change even with applying the magnetic field. So the correct value of the percolative length remains unachivable with this method. Thus, by assuming Eq. (18) we account for the specific feature of the ND technique and suppose a continuous growth of the cluster appearing at the local temperature $T_{C}^{\prime}$.

Thus, we fixed $\left\langle T_{C}\right\rangle$ and $\Delta T_{C}$ in $\rho\left(T_{C}\right)$ at the values obtained from the fit of the rotation angle $\phi$ for $H=10 \mathrm{G}$. Thereby we obtained satisfactory fits for the two parameters which are still unknown: $B_{0}^{2} R_{d}(0)$ and $\Delta$. From the best fit procedure we find $\Delta=0.65 \pm 0.05$ and $B_{0}^{2} R_{d}(0)=(4.11$ $\pm 0.01) \times 10^{4} \mathrm{G}^{2} \mathrm{~cm}$. From the parameter $B_{0}^{2} R_{d}(0)$ one can roughly estimate the value $R_{d}(0)$ substituting $B_{0}$ taken from the Table I at $H=10 \mathrm{G}: B_{0}=4620 \mathrm{G}$. Then we calculate easily the value $R_{d}(0)=2 \times 10^{5} \AA$ that is close to the domain size far below the transition temperature. The value of $R_{d}$ at the room temperature equals $8 \times 10^{4} \AA$ which coincides with the domain size in the invar alloys given by the multiple SANS. ${ }^{21}$ From Eq. (18) the correlation length of the disorder is estimated through the size of the magnetic inhomogeneities at the critical range $R_{0} \sim R_{d}(0) \tau_{1}^{\Delta} \approx 10^{4} \AA$.

In spite of the discrepancy between the supposition Eq. (18) and the percolative concept in Refs. 5-7, we agree with the statement that the ratio of the depth of the $T_{C}$ variations $\left(\Delta T_{C} /\left\langle T_{C}\right\rangle\right)$ and its characteristic correlation length $R_{0} / a$ ( $a$ is the lattice constant) determines the scenario of the transition: "percolative" or "homogeneous." For the case of FeNi alloy under study the criterium $R_{0} / a$ $\gg\left(\Delta T_{C} /\left\langle T_{C}\right\rangle\right)^{-2 / 3}$ is well fulfilled and, therefore, the percolative scenario is realized. 
It is worth to notice that the idea of the convolution of the power-law description with Gaussian distribution of transition temperature becomes commonly used to treat the experimental data in disordered systems. ${ }^{22-24}$ In our work we emphasize that the idea of $T_{C}$ distribution is closely related to enhancement of the large scale inhomogeneities above $T_{C}$ and therefore with the percolative scenario of the transition.

\section{SUMMARY}

The magnetic phase transition in a disordered system $\mathrm{Fe}_{70} \mathrm{Ni}_{30}$ was investigated. The experimental data of the neutron depolarization and SANS show a smeared phase transition. To interpret the data we apply the model where the Curie temperature is fluctuating around the average value $\left\langle T_{C}\right\rangle$. In this model the local $T_{C}$ variations are described by the disorder parameters of the system: $\left\langle T_{C}\right\rangle$, a spread of $T_{C}$ variations $\Delta T_{C}$, and the characteristic size of the local areas $R_{0}$, where $T_{C}$ variations occur [Eq. (1)]. The parameters of the transition obtained from the data are $\left\langle T_{C}\right\rangle=397$ $\pm 0.5 \mathrm{~K}, \Delta T_{C}=4.55 \mathrm{~K}$, and $R_{0} \sim 10^{4} \AA$. This means, ac- cording to the criterion proposed in Refs. 5-7 that the criterion $R_{0} / a \gg\left(\Delta T_{C} /\left\langle T_{C}\right\rangle\right)^{-2 / 3}$ is fulfilled in our disordered system, so the transition goes in accordance with the "percolative" scenario. In this case the percolation means that the locally ordered magnetic regions grow in the paramagnetic phase and form a large percolative cluster. It was also found that the magnetic cluster size $R_{d}$ grows with the local reduced temperature $\tau$ as $R_{d} \sim \tau^{\Delta}$ with $\Delta=0.65 \pm 0.05$. The reasons of the $T_{C}$ variations which result in smearing of the transition are still not clear. Nevertheless, the enhancement of the $T_{C}$-variations is proved by the ND experiments along with SANS and the parameters of the phase transition are found from the data.

\section{ACKNOWLEDGMENTS}

The authors would like to thank NWO (Dutch Organization of Advancement of Science) for providing funding to strengthen their cooperation. The work was also supported by RFFR (Projects No. 00-15 -96814 and 01-02-17286) and the Russian State Program "Neutron Research of Solids."
*Email address: grigor@rvv.lnpi.spb.su

${ }^{1}$ S.R. Andrews, J. Phys. C 19, 372 (1986).

${ }^{2}$ R.A. Cowley, Phys. Scr. 66, 24 (1996).

${ }^{3}$ S.V. Grigoriev, S.V. Maleyev, V.V. Runov, and A.I. Okorokov, Phys. Rev. B 58, 3206 (1998).

${ }^{4}$ S.V. Grigoriev, S.A. Klimko, S.V. Maleyev, A.I. Okorokov, V.V. Runov, and D.Yu. Chernyshov, Zh. Éksp. Teor. Fiz. 112, 2134 (1997) [JETP 85, 1168 (1997)].

${ }^{5}$ S.L. Ginzburg, Zh. Éksp. Teor. Fiz. 73, 1961 (1977) [Sov. Phys. JETP 46, 1029 (1977)].

${ }^{6}$ A.L. Korzhenevskii, H-O. Heuer, and K. Herrmanns, J. Phys. A 31, 927 (1998).

${ }^{7}$ A.L. Korzhenevskii, K. Herrmanns, and H-O. Heuer, Europhys. Lett. 45, 195 (1999).

${ }^{8}$ O.K. Antson, A.P. Bulkin, P.E. Hiismaki, T.K. Korotkova, V.A. Kudryashev, H.S. Kukkonen, V.G. Muratov, H.O. Poyry, A.F. Shchebetov, A.T. Tiitta, V.A. Trunov, and V.A. Ul'yanov, Physica B 156-157, 567 (1989).

${ }^{9}$ V.L. Sedov, Antiferro-magnetism of $\gamma$-iron. The Invar Problem (Moskow, Nauka, 1987).

${ }^{10}$ S.V. Maleyev and V.A. Ruban, Zh. Éksp. Teor. Fiz. 62, 416 (1972) [Sov. Phys. JETP 35, 222 (1972)].

${ }^{11}$ S.V. Maleyev, J. Phys. C 7, 23 (1982).

${ }^{12}$ R. Rosman and M.Th. Rekveldt, Phys. Rev. B 43, 8437 (1991).

${ }^{13}$ R. Rosman and M.Th. Rekveldt, J. Magn. Magn. Mater. 95, 319
(1991).

${ }^{14}$ S.V. Grigoriev, W.H. Kraan, S.V. Maleyev, A.I. Okorokov, M.Th. Rekveldt, and V.V. Runov, J. Neutron Res. 8, 155 (2000).

${ }^{15}$ S.V. Grigoriev, V.V. Runov, A.I. Okorokov, O.A. Gubin, G.P. Kopitsa, A.D. Tretiakov, and M.K. Runova, Nucl. Instrum. Methods Phys. Res. A 389, 441 (1997).

${ }^{16}$ A.Z. Menshikov, S.K. Sidorov, and V.A. Shestakov, Zh. Éksp. Teor. Fiz. 70, 163 (1976) [Sov. Phys. JETP 43, 85 (1976)].

${ }^{17}$ T. Takeda, S. Komura, T. Miyazaki, Y. Endoh, and S. Itoh, J. Magn. Magn. Mater. 70, 431 (1987).

${ }^{18}$ M. Acet, E.F. Wassermann, K. Andersen, A. Murani, and O. Scharpff, Europhys. Lett. 40, 93 (1997).

${ }^{19}$ R.A. Cowley, G. Shirane, R.J. Birgeneau, E.S. Svensson, and H.J. Cuggenheim, Phys. Rev. B 22, 4412 (1980).

${ }^{20}$ G. Aeppli, S.M. Shapiro, R.J. Birgeneau, and H.S. Chen, Phys. Rev. B 28, 5160 (1983).

${ }^{21}$ Yu.G. Abov, D.S. Denisov, F.S. Dzheparov, N.O. Elutin, D.V. Lvov, S.K. Matveyev, Yu.I. Smirnov, and A.O. Eidlin, Zh. Éksp. Teor. Fiz. 114, 2194 (1998) [JETP 87, 1195 (1998)].

${ }^{22}$ K. Hirota, G. Shirane, Q.J. Harris, Q. Feng, R.J. Birgeneau, M. Hase, and K. Uchinokura, Phys. Rev. B 52, 15412 (1995).

${ }^{23}$ A.Yu. Romanov, V.P. Silin, and D. Wagner, Phys. Lett. A 226, 310 (1997).

${ }^{24}$ A.Yu. Romanov and V.P. Silin, Zh. Éksp. Teor. Fiz. 113, 213 (1998) [JETP 86, 120 (1998)]. 